\title{
BETA ENHANCEMENT BY RELATIVISTIC ELECTRON RINGS IN BUMPY TORI *
}

\author{
T. KAMMASH and K.T. NGUYEN \\ University of Michigan, Ann Arbor, MI 48109, USA
}

The maximum achievable beta (ratio of plasma pressure to magnetic pressure) value in a bumpy torus such as Elmo (EBT) is determined primarily by the stability of the system to the interchange modes. These modes have as their source of free energy the drifts of the hot electron component that arise from the curvature and gradients of the confining magnetic field. At low (compared to the ion gyro) frequencies the only modes that exist are the core plasma and hot electron interchange, but at frequencies near the ion cyclotron frequency, instabilities arising from coupling of the interchange and compressional Alfvén wave also appear. In most, if not all previous treatments of these instabilities, the hot electrons were treated as non-relativistic. In this paper we re-examine these modes using a relativistic formulation for the hot electrons which we also treat as highly anisotropic by neglecting their parallel momenta relative to the perpendicular momenta and their rest mass energy. We find a significant enhancement in the ion beta value as a result of treating the ring electrons relativistically. For typical present day EBT parameters we find that the value of the ion beta increases by about $50 \%$ as a result of increasing the hot electron mean energy from about several keV to few MeV. At such an energy the microwave power for heating these electrons is also optimum since the drag on the background plasma also reaches its minimum value.

\section{Analysis and dispersion equation}

Several investigations [1] have in recent years addressed the stability of EBT plasma to the interchange mode [2,3], and to the coupled interchange-compressional Alfvén mode [4,5], using the kinetic approach in a slab geometry and producing successively lower achievable core beta values. In all of these studies the ring-core interaction was taken into account but the hot electron species was treated non-relativistically. In this investigation we re-examine the stability of these modes in the presence of a relativistic hot electron component for which we write the Hamiltonian as

$$
H=\left(m^{2} c^{4}+p^{2} c^{2}\right)^{1 / 2}+f_{\mathrm{g}} x,
$$

where $m$ is the rest-mass of the electron, $p$ is the momentum, $c$ is the speed of light and $f_{\mathrm{g}}$ is the gravitational force associated with the magnetic field curvature. By virtue of their heating, these electrons are generally highly anisotropic, and we simulate this effect in eq. (1) by letting $\left\langle p_{z}^{2}\right\rangle \ll\left\langle p_{\perp}^{2}\right\rangle, m c^{2}$, where $p_{z}$ and $p_{\perp}$ are respectively the momenta parallel and perpendicular to the magnetic field, and $m c^{2}$ is the rest mass energy. With this modification eq. (1) becomes:

$$
H=\left(m^{2} c^{4}+p_{\perp}^{2} c^{2}\right)^{1 / 2}+f_{\mathrm{g}} x=\gamma m c^{2}+f_{\mathrm{g}} x,
$$

where the relativistic parameter $\gamma$ is given by

$$
\gamma=\left(1+\frac{p_{\perp}^{2}}{m^{2} c^{2}}\right)^{1 / 2}=\left(1-\frac{v_{\perp}^{2}}{c^{2}}\right)^{-1 / 2} .
$$

In a slab geometry where the external magnetic field is given by $B=B_{0}(1+\epsilon x) \hat{z}$ and in which the density gradient and the gravitational force are also in the $x$-direction, the constants of the motion are the

\footnotetext{
* Work supported by US DOE.
} 
above-mentioned Hamiltonian and the canonical angular momentum associated with the ignorable coordinate. In terms of these constants of motion the equilibrium distribution function for the hot electrons can be put in the form

$$
f_{0}\left(p_{z}, p_{\perp}^{2}, x_{\mathrm{g}}\right)=N_{0}\left(1+\epsilon^{\prime} x_{\mathrm{g}}\right) F\left(p_{z}\right)\left(\frac{\pi \beta}{2}\right)^{1 / 2} \frac{\mathrm{e}^{-\left(\beta \gamma+2 x / R_{\mathrm{c}}\right)}}{m^{2} c^{2} K_{3 / 2}(\beta)},
$$

where

$$
\begin{aligned}
& x_{\mathrm{g}}=x+\frac{c p_{y}}{e B_{0}} ; \quad \beta=m c^{2} / T_{\perp}, \\
& 1=\int F\left(p_{z}\right) \mathrm{d} p_{z}, \quad f_{\mathrm{g}}=\frac{2 m c^{2}}{R_{\mathrm{c}} \beta}=\frac{2 T_{\perp}}{R_{\mathrm{c}}},
\end{aligned}
$$

and $N_{0}, R_{\mathrm{c}}$ and $K_{3 / 2}(\beta)$ are the hot electron density, the radius of curvature, and the modified Bessel function respectively. It can readily be shown [1] that

$$
\epsilon^{\prime}=\frac{1}{L_{n}}+\frac{2}{R_{\mathrm{c}}}, \quad \epsilon=-\frac{\beta_{\mathrm{t}}}{2} \epsilon^{\prime}=\left(\frac{1}{B} \frac{\mathrm{d} B}{\mathrm{~d} x}\right),
$$

where $L_{n}$ is the density gradient scale length, and $\beta_{\mathrm{t}}$ is the total $\left(\beta_{\mathrm{e}}+\beta_{\mathrm{i}}+\beta_{\mathrm{h}}\right)$ beta of the system. In order to calculate the hot electron components of the dielectric tensor we first solve the linearized Vlasov equation, namely

$$
f_{1}=e \int_{-\infty}^{t} \mathrm{~d} t^{\prime}\left[\boldsymbol{E}+\frac{\boldsymbol{v} \times \boldsymbol{B}}{c}\right] \cdot \frac{\partial f_{0}}{\partial \boldsymbol{p}^{\prime}}
$$

where the integration is over the unperturbed particle orbits. For the relativistic hot electrons these orbits have the form

$$
\begin{aligned}
& x=\frac{\gamma v_{\perp}}{\Omega} \cos \left(\theta-\frac{\Omega}{\gamma} t\right)-\frac{\gamma v_{\perp}}{\Omega} \cos \theta, \\
& y=-\frac{\gamma v_{\perp}}{\Omega} \sin \left(\theta-\frac{\Omega}{\gamma} t\right)+\frac{\epsilon v_{\perp}^{2} \gamma t}{2 \Omega}+\frac{f_{\mathrm{g}} t}{m \Omega}+\frac{\gamma v_{\perp}}{\Omega} \sin \theta,
\end{aligned}
$$

where the gyrofrequency, $\Omega=e B_{0} / m c$, is expressed in terms of the rest mass. Following a standard but lengthy procedure we calculate the hot electron components of the dielectric tensor to be

$$
\begin{aligned}
\epsilon_{\|}^{\mathrm{h}}= & \left(k \lambda_{\mathrm{Di}}\right)^{-2} \frac{\delta}{\tau_{\mathrm{h}}}\left[1+\frac{2\left(\omega-\omega_{*_{\mathrm{h}}}\right)}{\omega_{B \mathrm{~h}}} \frac{\beta}{1+\beta}\left\{\frac{1}{\beta}+\frac{1}{\Delta-\alpha}\left(\mathrm{e}^{-G}(a \alpha+1) E_{\mathrm{i}}(G)-\mathrm{e}^{-Q}(a \Delta+1) E_{\mathrm{i}}(Q)\right)\right\}\right] \\
& +\frac{\delta}{\tau_{\mathrm{h}}}\left(k \lambda_{\mathrm{Di}}\right)^{-2} \cdot \text { FLR }
\end{aligned}
$$

with the finite Larmor radius term (FLR) given by

$$
\begin{aligned}
\text { FLR }= & -\frac{\left(k \rho_{\mathrm{eh}}\right)^{2}}{2} \frac{\left(\omega-\omega_{* \mathrm{~h}}\right)}{\omega_{B \mathrm{~h}}} \frac{\beta^{2}}{1+\beta}\left\{\frac{1+a}{\beta}+\frac{1}{\beta^{2}}+\frac{1}{\Delta-\alpha}\left[\mathrm{e}^{-G}\left(a^{2} \alpha+a\right) E_{\mathrm{i}}(G)\right.\right. \\
& \left.\left.-\mathrm{e}^{-Q}\left(a^{2} \Delta+a\right) E_{\mathrm{i}}(Q)\right]\right\} .
\end{aligned}
$$

In addition we have

$$
\begin{aligned}
\epsilon_{22}^{\mathrm{h}}= & \frac{\beta_{\mathrm{h}}}{4}\left(\frac{\left(\omega-\omega_{* \mathrm{~h}}\right)}{\omega_{B \mathrm{~h}}}\right) \frac{\beta^{3}}{1+\beta}\left\{\frac{(1+a) a}{\beta}+\frac{2+a}{\beta^{2}}+\frac{2}{\beta^{3}}\right. \\
& \left.+\frac{1}{\Delta-\alpha}\left[\mathrm{e}^{-G}\left(a^{3} \alpha+a^{2}\right) E_{\mathrm{i}}(G)-\mathrm{e}^{-Q}\left(a^{3} \Delta+a^{2}\right) E_{\mathrm{i}}(Q)\right]\right\}
\end{aligned}
$$




$$
\begin{aligned}
\epsilon_{12}^{\mathrm{h}}= & \left(k \lambda_{\mathrm{Di}}\right)^{-1}\left(\frac{\beta_{\mathrm{i}}}{2}\right)^{1 / 2} \frac{\delta \beta^{2}}{1+\beta}\left\{\frac{1+a}{\beta}+\frac{1}{\beta^{2}}+\frac{1}{\Delta-\alpha}\left[\mathrm{e}^{-G}\left(a^{2} \alpha+a\right) E_{\mathrm{i}}(G)-\mathrm{e}^{-Q}\left(a^{2} \Delta+a\right) E_{\mathrm{i}}(Q)\right]\right\} \\
& \times\left(\frac{\omega-\omega_{* \mathrm{~h}}}{\omega_{B \mathrm{~h}}}\right) .
\end{aligned}
$$

In the above expressions $\lambda_{D i}$ denotes the ion Debye length, $E_{\mathrm{i}}(G)$ the familiar exponential integral, $\omega_{*}$ the diamagnetic drift frequency, $\omega_{B \mathrm{~h}}$ is the drift frequency associated with the magnetic field gradient, $\omega_{\mathrm{gh}}$ is the drift frequency associated with the field curvature, and

$$
\begin{aligned}
& a=\frac{2\left(\omega-\omega_{\mathrm{gh}}\right)}{\beta \omega_{B \mathrm{~h}}} ; \quad \alpha=\frac{a}{2}-\frac{1}{2}\left(a^{2}+4\right)^{1 / 2} ; \quad \Delta=\frac{a}{2}+\frac{1}{2}\left(a^{2}+4\right)^{1 / 2} ; \quad G=\beta(\alpha-1) ; \\
& Q=\beta(\Delta-1) ; \quad \beta=\frac{m c^{2}}{T_{\perp \mathrm{h}}} ; \quad \tau_{\mathrm{h}}=\frac{T_{\perp \mathrm{h}}}{T_{\mathrm{ci}}} ; \quad \delta=\frac{N_{\mathrm{h}}}{N_{\mathrm{i}}}=\frac{\beta_{\mathrm{h}}}{\tau_{\mathrm{h}} \beta_{\mathrm{i}}} ; \quad \tau_{\mathrm{e}}=\frac{T_{\mathrm{ce}}}{T_{\mathrm{ci}}} .
\end{aligned}
$$

When combined with the dielectric tensor components $\epsilon_{i j}^{\mathrm{e}}, \epsilon_{i j}^{\mathrm{i}}$ of the background plasma the above results yield a dispersion equation which can be expressed as

$$
D_{\mathrm{es}} D_{\mathrm{em}}+(c T)^{2}=0
$$

where

$$
\begin{aligned}
& D_{\mathrm{es}}=1+\epsilon_{\|}^{\mathrm{i}}+\epsilon_{\|}^{\mathrm{e}}+\epsilon_{\|}^{\mathrm{h}} \simeq \epsilon_{\|}^{\mathrm{i}}+\epsilon_{\|}^{\mathrm{e}}+\epsilon_{\|}^{\mathrm{h}}, \\
& D_{\mathrm{em}}=1-\left(\epsilon_{22}^{\mathrm{i}}+\epsilon_{22}^{\mathrm{e}}+\epsilon_{22}^{\mathrm{h}}\right), \\
& c T=\epsilon_{12}^{\mathrm{i}}+\epsilon_{12}^{\mathrm{e}}+\epsilon_{12}^{\mathrm{h}} .
\end{aligned}
$$

The expressions for $\epsilon_{i j}^{e}$ and $\epsilon_{i j}^{i}$ have been obtained for Maxwellian distributions elsewhere [4,5], but modified here to account for the "phase" effect in $x_{\mathrm{g}}$ (see eq. (5)) which plays an important role in the coupled-mode instability at $\omega \simeq \Omega_{\mathrm{i}}$. The dispersion eq. (14) is investigated numerically for the stability boundary due to the multiplicity of the free parameters which are $\beta, \beta_{\mathrm{h}}, \beta_{\mathrm{i}}, \rho_{\mathrm{i}} / L_{\mathrm{n}} k \rho_{\mathrm{i}}, L_{n} / R_{\mathrm{c}}, \tau_{\mathrm{h}}$ and $\tau_{\mathrm{e}}$.
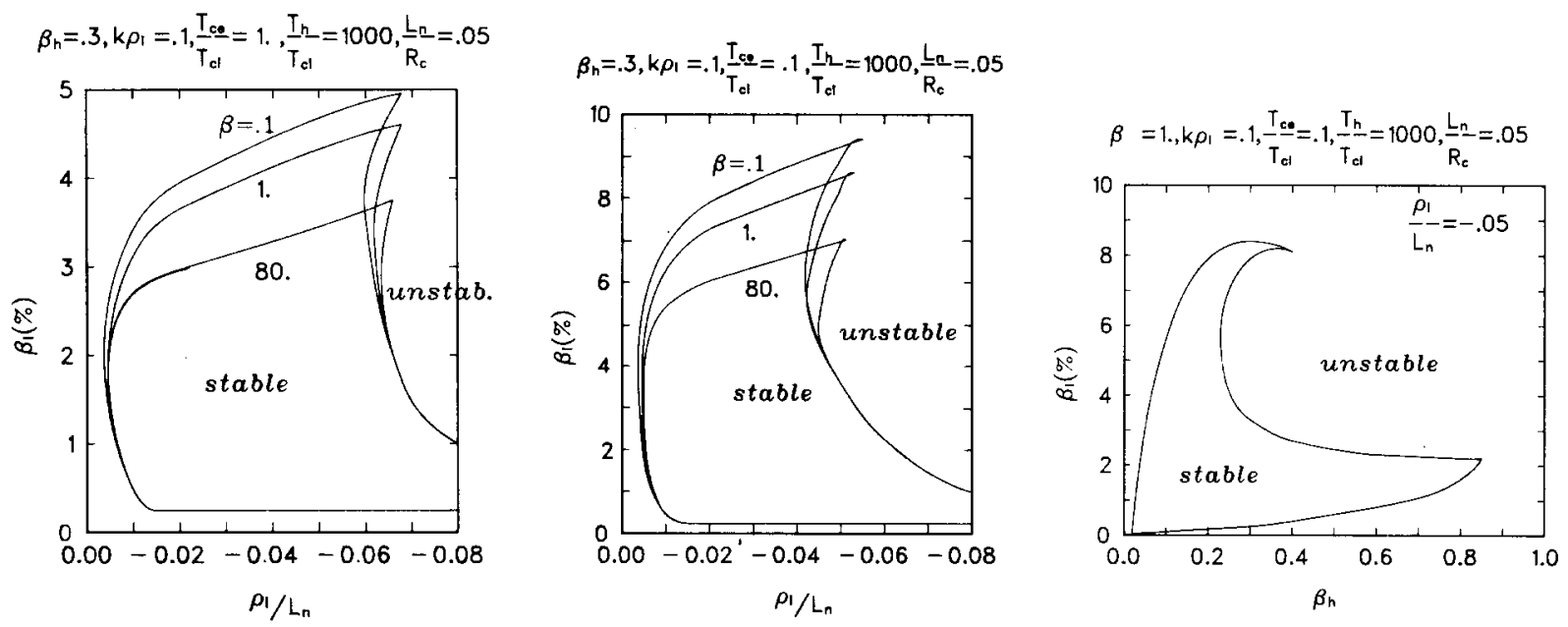

Fig. 1. Enhancement of the ion beta as a result of increasing $\beta$ from 80 to 0.1 .

Fig. 2. Improvement in the ion beta as a result of decreasing the background electron temperature relative to that of the ions.

Fig. 3. Change in the stability window resulting from the coupled interchange-Alfven modes. 


\section{Results}

Significant improvement in the value of $\beta_{\mathrm{i}}$ occurs when the ring electrons are treated relativistically as demonstrated in fig. 1 . The lowest curve $\left(\beta=m c^{2} / T_{\perp \mathrm{h}} \simeq 80\right)$ represents the non-relativistic result which for the parameters shown agree with those of refs. 2 and 3 . The most dramatic enhancement in $\beta_{\mathrm{i}}$ of about $50 \%$ occurs at ring electrons mean energy of about two $\mathrm{MeV}$, beyond which the improvement is not substantial. At this energy the microwave power needed to heat these electrons is also optimum since the drag reaches its minimum value at this energy [6]. Although the core beta does not change much, additional increase in $\beta_{\mathrm{i}}$ can be achieved if the temperature of the ions is increased relative to that of the background electrons as illustrated in fig. 2 (and contemplated in EBT-P). Current experiments with hot electron temperature of about $400 \mathrm{keV}$ reveal [7] a distribution function of a non-relativistic Maxwellian form. In terms of the present analysis this seems to suggest that the dynamics of the realitivistic electrons, rather than the precise form of the distribution function, is perhaps the major factor responsible for the beta enhancement. Although increasing $\beta_{\mathrm{h}}$ has a stabilizing effect on the interchange mode, fig. 3 also shows that due to the coupled mode there exists a window in $\beta_{h}$ which defines a channel of stable region in which multiple values of $\beta_{\mathrm{i}}$ exist. This window and the corresponding channel become narrower as $\rho_{\mathrm{i}} / L_{n}$ increases. Finally, this analysis reveals that the stability boundary of the interchange mode is particularly sensitive to the hot electron model. A delta function representation of these electrons yields about twice as high a maximum $\beta_{\mathrm{i}}$ as does a Maxwellian representation in agreement with the predictions of Nelson [2].

\section{References}

[1] D.A. Spong, Oak Ridge Report ORNL/TM-8021 (October 1981).

[2] D.B. Nelson, Phys. Fluids 23 (1980) 1850.

[3] J.W. VanDam and Y.C. Lee, Proc. Workshop on EBT ring physics (Oak Ridge, CONF-791228, Dec. 1979) p. 471.

[4] D.A. Spong and A. El-Nadi, Bull. Am. Phys. Soc. 25 (1980) 964.

[5] K.T. Tsang and C.Z. Cheng, Proc. Workshop on EBT stability theory (Oak Ridge, CONF-81052, May 1981) p. 141.

[6] S.K. Borowski, N.A. Uckan, E.F. Jeager and T. Kammash, Proc. Workshop on EBT ring physics (Oak Ridge, CONF-791228, Dec. 1979) p. 319.

[7] G.R. Haste, private communication. 